\title{
ENERGY CONSUMPTION REAL TIME MONITORING IN INTERNET OF THINGS CONTEXT
}

\author{
Răzvan Cristian MARALES \\ Bucharest University of Economic Studies, Romania \\ razvan.marales@gmail.com \\ Osman Bülent TÖR \\ Bucharest University of Economic Studies, Romania \\ osman.tor@epra.com.tr
}

\begin{abstract}
This paper is making an in depth presentation of energy consumption analyze and monitoring in real time and near real time within Internet of Things conditions. Concepts such as energy consumption, Demand Side Management, real time and near real time monitoring, Internet of Things and concrete tools for this kind of an implementations will be described. The main purpose of the paper is to describe the context of a real-life situation, where the monitoring of energy consumption in real time represent a challenge which may bring in the end noticeable benefits and propose the architecture and technologies for resolving such of a challenge.
\end{abstract}

Keywords: Energy consumption, Internet of Things (IoT), Demand Side Management (DSM), real time monitoring

JEL classification: L86, D80, Q41

DOI: $10.12948 / \mathrm{ie} 2019.03 .05$

\section{Introduction}

Demand Side Management (DSM) involves several methods such as monitoring and control of electric appliances, consumption optimization, forecast, customer segmentation, consumers behaviour and profiles. These methods should be based on consideration of comfort levels of the consumers. For example, lighting loads cannot be taken as a control parameter during evening times just to save money from electric bills. However, operation of washing machines, for example, can be scheduled to mid-night, when the electric tariff is minimum. Requirement for comfort level is subjective and differs from one person to another. Therefore, consumption optimization should take into account the comfort level of the relevant consumer. According to studies in the literature, savings from the electricity bills can reach up to $50 \%$ if they shift their controllable loads to off-peak periods when the tariff is one third of the peak tariff [1]. Recent developments in battery storage technologies show that battery storage devices can be a promising solution in consumption optimization problem. Battery storage devices contributes to the comfort level of consumers while minimizing the electric bills [2]. The authors in [2] proposed a method to determine the optimum capacity of a storage device not only for electricity bill minimization of consumers but also consumption peak minimization enabling minimizing technical losses in the grid and delaying grid onerous investments. Storage device is a control parameter in the consumption optimization problem. Differently from controlling electric devices, like washing machine, storage devices can be controlled in two directions: 1) generation mode (discharging); 2) demand mode (charging).

In a smart grid and IoT context, the existence of dynamic tariffs and bidirectional communications simultaneously allow and require an active role from the end-user concerning 
consumption optimization [3]. Utilities are now focusing more on DSM methods than before, thanks to developments in two-way communication between individual user devices and the electricity provider. Time-of-use tariff (time-varying prices) is the simplest and the most traditional DSM approach to minimize peak load in the system. Smart grids are capable of twoway communication between individual user devices and the electricity provider, enabling providers to create a control-feedback loop using time-dependent pricing [4]. Energy management systems are required to monitor consumption/generation/storage and to make the best decisions according to input signals and the user's needs and preferences. Design of consumption optimization algorithms to be implemented in those systems require the prior characterization of domestic electricity demand and categorization of loads, according to availability, typical usage patterns, working cycles and technical constraints. The authors in [3] presented a characterization of household electricity consumption and they proposed an operational categorization of end-use loads. This study is important in the sense of categorizing demands of consumers as control parameters. Such a categorization is important to model consumption optimization algorithm. The authors in [5] investigated a household electricity segmentation methodology that uses an encoding system with a pre-processed load shape dictionary. The authors satisfactorily segmented household energy consumption using hourly data. Although main scope of mechanisms such as time-varying prices is to encourage consumers to reduce their consumption during high electricity demand, it is usually a hassle to residential customers to manually adjust their loads in response to dynamic electricity prices. In [6], the authors present a study from a pilot project involving smart metering, remote load control, pricing based on the hourly spot price combined with a time of day network tariff, and a token provided to the customers indicating peak hours. It is observed in the pilot study that demand response is around $1 \mathrm{kWh} / \mathrm{h}$ for customers with standard electrical water heaters. This capacity is addressed as a potential load reduction in the day ahead market from a market perspective. DSM methods and their effects in electricity market prices have been given interest indeed recently by several authors in the literature. In [7], definition and classification of DSM methods are presented by addressing potential benefits and associated cost components. In addition, the authors highlighted the most common indices used for DSM impact measurement and evaluation. Experiences of some utilities which implement different DSM methods are also discussed in the study. In [8], the authors presented a pilot study of DSM from household customers utilizing smart metering, remote load control and pricing based on the hourly day ahead market price combined with a time of day network tariff. In this paper we present an architecture for implementing DSM methods for real-time monitor and control of the IoT appliances. The proposed architecture is based on Big Data technologies and allows the consumers and the electricity supplier to interactively visualize the consumption data.

\section{Architecture and implementation}

As shown in Figure 1, the architecture of the system is split in severals components and entities, serving different scopes. From the scope point of view, the components can be separated in three parts. First part is where data is created and collected, which contains the end devices and data collector devices that are places physical in the end consumers apartments together with the community data collector that live inside the community and it has a fix number of apartment which relate with.

Second one is the transportation point, here the data that was collected will be transported from the physical places (apartments and communities) to a more centralized place in order to be monitor and interpreted. This component is the transportation channel. 
In the end, the third part is describe as the processing and monitoring section, here the real time and near real time processing of the collected data have place. Contains the real time persistence component and the real time monitoring engine that providing parallel data processing for monitoring purposes.

At component level, each unit is designed to respect some elementary rules and achieve different purposes, decoupled one from each other. The advantages will be seen when all the components are integrated together as a system that can provide a solution for real time monitoring of energy consumption.

The end devices can be described as smart or intelligent hardware, that is placed in end consumers apartments or houses, being able to calculate the energy consumption in real time and expose it using a well known interface. This devices are usually home appliance such as washing machines, ovens, AC units or smart meters devices, smart plugs, smart bulbs. They represent the starting point of data creation, is vital that the information expose by this kind of devices is well calibrated and correct.

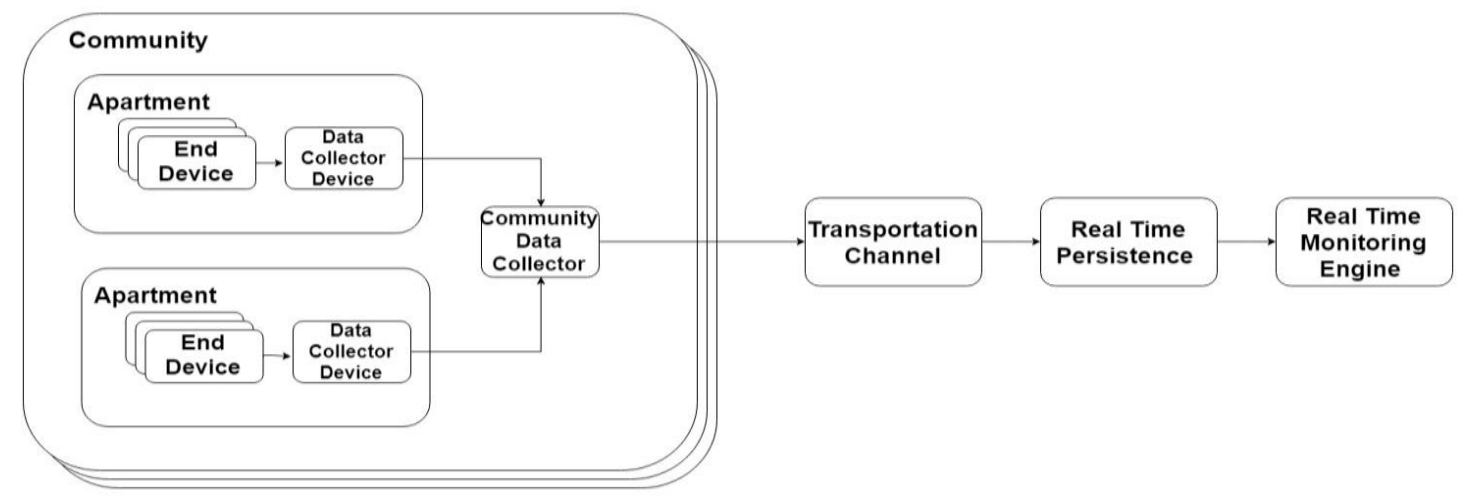

Figure 1. Overall architecture

Data collector device is designed as middleware between apartments devices and community system. The main purpose of it is to read and collect all the data created by end devices about the energy consumption, make filtration and conversation on it and push it outside the apartments to the interested systems, in this case the community data collector. For this kind of devices the most suitable implementation are small PC or intelligent small controllers, that doesn't consume a big amount of resources, has the possibility to integrate using different interfaces and can communicate with the community data collector. It is vital for a data collector device to know as much as possible interfaces and protocol for smart home communication, because of market variety, the end devices manufacturers are providing a various list of interfaces, starting from simple Wi-Fi connection or Bluetooth to more specific protocols like ZigBee or Z-Wave.

Community data collector is the component that interact with all data collectors inside the apartments by reading the created data and transporting it outside the community, into a bigger system. This component add a real benefit to the overall architecture, by providing a central point within community where data is collected, this can be helpful in case of failovers outside the community, in this cases the data can be cache and transfer after the issue is remediated, also as future feature this component can work intelligent, being able to monitor all the apartments in the community and take smart decision inside it, without needing to propagate all the information outside of community.

The transportation channel is the first component that lives outside the community, it interact with the community data collector, receiving the data created inside community and propagate it to the interested parties. Is important that this component has two core features, asynchronous 
communication and scalability. Being in the middle of the communication chain, should not have blocking operations, this is the main argument for mandatory request of having asynchronous communication. Same reasoning is used for the scalability, being in the middle the components needs to provide a way to increase the resources and process the same input of data even if is under high or low load.

Real time persistence component is used to cache the data related with the energy consumption receive from the transportation channel. The persistence service should provide an optimal and fast mechanism of writing and reading data, to ensure that the processing goes as fast as possible, with minimum delays, bringing the monitoring to real time or near real time.

After transporting the data in a fast way and persist it by the other components, the real time monitoring engine is coming in place. Its main purpose is to read the data for a time frame (for a more accurate monitoring the data frames should be small, between 30 seconds and 1 minute) and apply different algorithms to it in order to extract core information that will be use in decision making. Furthermore, the information extracted will enable the possibility of proactive measures and actions. A various list of algorithms may be applied on read data, starting with some simple statistical algorithms such as average or median at apartments or communities level to more complex algorithms for forecasting and trends recognition.

\section{Implementation}

For implementation we decided to simulate a real life system, which consist of a couple of apartments and communities. Trying to be as close as possible to reality, data was simulated for a time frame of 3 months. As end devices we used smart plugs from TP-LINK, model HS110. The plug is capable to read in real time the energy consumption information and expose it through a local WI-Fi connection using HTTP protocol. Using smart plugs provide a real advantage, each home appliance can be monitored without the prerequisite of being smart.

For data collector device a Raspberry Pi model 3 is used, which is a small controller, with its own processor, memory and WI-Fi adaptor that give the possibility to interact with the smart plug in a very convenient way. The controller is running a small distribution of Linux operating system and can execute different small applications continuously. To make sure that the data is pulled in real time we wrote a NodeJs application which query at an interval of seconds the smart plugs and read the energy consumption information. Furthermore, the small controller is pushing all the data to the community data collector.

The community data collector is created as a standalone Java server side application, which is exposing an intelligent REST API. The API is consumed by the Raspberry PI NodeJs application, and represent the mode in which the two entities are integrated, able to communicate and exchange information.

All the components described previously where place physical into apartments or nearby, to achieve a faster communication locally. Forwards, all the component that will help to centralizate date higher than community level will be place in a public cloud, this will permit to provide a bigger flexibility and scalability of the resources and applications.

Next component is the transportation channel, which is implemented using a message queue open source project from Apache Foundation named Apache Kafka. The queue is working under the publish subscribe pattern, in our case the publishers are the community data collectors, that are pushing gathered data into the queue, and the subscribers will be the components that are interested in this data, the real time persistence. We created the configurations to have for each community a separate topic in the queue, in this way the data will be decoupled from community to community and it will be easy to differentiate. Majority of the monitoring will be done on community or apartment level, but for cases where we aim 
for a bigger overview, as example the consumption of all communities in real time, the real time monitoring engine will be able to bring together all the topics and provide this information. As real time persistence solution a cluster of Elasticsearch is used. The data which is transferred between Kafka queues and Elasticsearch will be done automatically using Logstash, a small agent that is able to integrate this two components without too many requirements, using just a configuration file. Logstash brings a real advantage into the flow, cutting the transportation overheads. Keeping the pattern, as implemented for Kafka topics, each community will own separate Elasticsearch indexes. The Elasticsearch cluster was chosen as implementation for the real time persistence component because is providing a suitable solution for fast reads and writes, brings data index capabilities and furthermore is able to provide mechanisms of aggregations on data on the flight.

For real time monitoring engine we decide to use Apache Spark, having one master that is coordinating three workers. As application example in Listing 1 can be check, which show a sample of code from our Spark job.

More comprehensive, the Spark job is scheduler to start periodically and query the Elasticsearch index in order to receive data about energy consumption at time frame level. The information will be fetch as a RDD, which is a specific way of represent data set using Spark. This data set will be map to a more easy to use structure, that will contain just the apartment id and device consumption. Each item will be linked with a value, in our case 1, this values will be accumulated as a count.

In the next stage all items will be group by apartment id. Two values will be calculated, first value being the total consumption per apartment and the second the total count of items per apartment. This values will reduce to a final result, which will return the average as total amount of consumption divided by object count, all group per apartments. For an easy understanding the results are printed to the console.

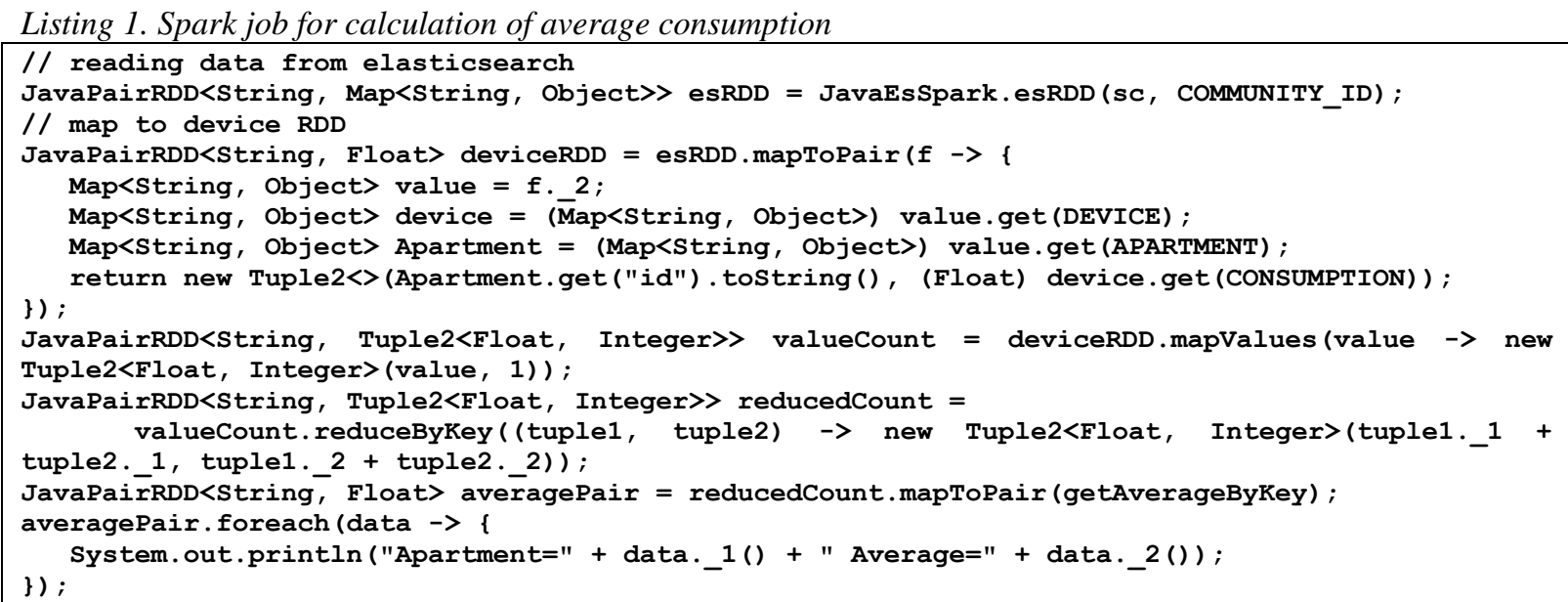

We decided that Spark is a suitable solution for this kind of monitoring because it provides parallel processing at workers level, which is a powerful feature for rapid calculations of algorithms. Spark jobs are running once at 30 seconds, calculate the energy consumption metrics and save the results back to Elasticsearch. From Elasticsearch the data is visualized using Kibana, a user interface tool that is implemented over Elasticsearch database. In Figure 2 the final result of the Spark job that is processing the energy consumption average can be seen. The result is presented on a map, that is pointing out the apartments location together with the final metrics. 


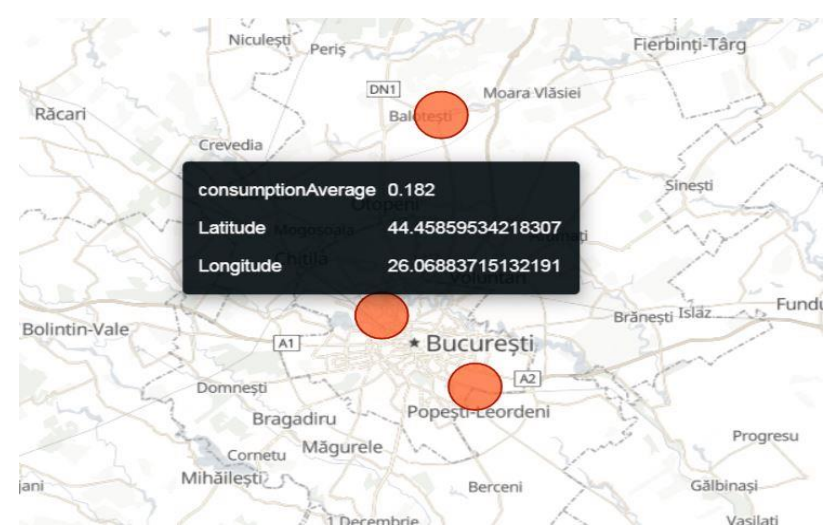

Figure 2. Spark job for calculation of average consumption

\section{Conclusions}

As DSM method, real time and near real time monitoring of energy consumption within an IoT context is a broad topic and we manage in this paper to present a theoretical and practical overview of the topic, but the subject can be further study. As future studies, we aim to investigate the possibility of moving a big piece of monitoring from the public cloud to community level, as infrastructure and logical components, which may bring costs and efficiency advantages in order to apply more DSM methods such as optimization and forecasting.

\section{Acknowledgment}

This paper presents the scientific results of the project "Intelligent system for trading on wholesale electricity market" (SMARTRADE), co-financed by the European Regional Development Fund (ERDF), through the Competitiveness Operational Programme (COP) 2014-2020, priority axis 1 Research, technological development and innovation (RD\&I) to support economic competitiveness and business development, Action 1.1.4 - Attracting high-level personnel from abroad in order to enhance the RD capacity, contract ID P_37_418, no. 62/05.09.2016, beneficiary: The Bucharest University of Economic Studies.

\section{References}

[1] A. Liu T, Chen S, Liu Y, Xu Z, Che Y, Duan Y, "SHE: Smart home energy management system for appliance identification and personalized scheduling". in ACM International Joint Conference on Pervasive and Ubiquitous Computing; 13-17 September 2014; Seattle, WA, USA. New York, NY, USA: ACM. pp. 247-250.

[2] S.V.Oprea, A.Bara, M.E.Cebeci, O.B.Tör, "Promoting peak shaving while minimizing electricity consumption payment for residential consumers by using storage devices," Turkish Journal of Electrical Engineering \& Computer Sciences, 2017 (25) 3725-3737.

[3] A. Soares, Á. Gomes, and C. H. Antunes, "Categorization of residential electricity consumption as a basis for the assessment of the impacts of demand response actions," Renew. Sustain. Energy Rev., 2014.

[4] C. Joe-Wong, S. Sen, S. Ha, and M. Chiang, "Optimized day-ahead pricing for smart grids with device-specific scheduling flexibility,” IEEE J. Sel. Areas Commun., 2012.

[5] J. Kwac, J. Flora, R. Rajagopal, "Household energy consumption segmentation using hourly data", IEEE Trans. Smart Grid, vol. 5, no. 1, pp. 420-430, Jan. 2014.

[6] H. Saele, O. S. Grande, "Demand Response from Household Customers: Experiences From a Pilot Study in Norway," IEEE Transactions on Smart Grid, vol. 2, no. 1, March 2011.

[7] M. H. Albadi, E. F. El-Saadany, "A summary of demand response in electricity markets", Elect. Power Syst. Res., vol. 78, no. 11, pp. 1989-1996, 2008.

[8] O. S. Grande, I Graabak, I. Vognild, A. H. Wilberg, "Market based Demand Response. End User involvement and experiences from Norwegian Pilots." CIRED, Vienna, Austria, 2007. 